This is the author's final, peer-reviewed manuscript as accepted for publication. The publisher-formatted version may be available through the publisher's web site or your institution's library.

\title{
Forecasting cosmological parameter constraints from near- future space-based galaxy surveys
}

Anatoly Pavlov, Lado Samushia, and Bharat Ratra

\section{How to cite this manuscript}

If you make reference to this version of the manuscript, use the following information:

Pavlov, A., Samushia, L., \& Ratra, B. (2012). Forecasting cosmological parameter constraints from near-future space-based galaxy surveys. Retrieved from http://krex.ksu.edu

\section{Published Version Information}

Citation: Pavlov, A., Samushia, L., \& Ratra, B. (2012). Forecasting cosmological parameter constraints from near-future space-based galaxy surveys. The Astrophysical Journal, 760(1).

Copyright: (c) 2012. The American Astronomical Society.

Digital Object Identifier (DOI): doi:10.1088/0004-637X/760/1/19

Publisher's Link: http://iopscience.iop.org/0004-637X/760/1/19/

This item was retrieved from the K-State Research Exchange (K-REx), the institutional repository of Kansas State University. K-REx is available at http://krex.ksu.edu 
KSUPT-12/3 June 2012

\title{
Forecasting cosmological parameter constraints from near-future space-based galaxy surveys
}

\author{
Anatoly Pavlov ${ }^{1}$, Lado Samushia ${ }^{2}$, and Bharat Ratra ${ }^{1}$
}

\begin{abstract}
The next generation of space-based galaxy surveys are expected to measure the growth rate of structure to about a percent level over a range of redshifts. The rate of growth of structure as a function of redshift depends on the behavior of dark energy and so can be used to constrain parameters of dark energy models. In this work we investigate how well these future data will be able to constrain the time dependence of the dark energy density. We consider parameterizations of the dark energy equation of state, such as XCDM and $\omega$ CDM, as well as a consistent physical model of time-evolving scalar field dark energy, $\phi$ CDM. We show that if the standard, specially-flat cosmological model is taken as a fiducial model of the Universe, these near-future measurements of structure growth will be able to constrain the time-dependence of scalar field dark energy density to a precision of about $10 \%$, which is almost an order of magnitude better than what can be achieved from a compilation of currently available data sets.
\end{abstract}

\section{Introduction}

Recent measurements of the apparent magnitude of Type Ia supernovae (SNeIa) continue to indicate, quite convincingly, that the cosmological expansion is currently accelerating (see, e.g., Conley et al. 2011; Suzuki et al. 2012; Li et al. 2011; Barreira \& Avelino 2011).

If we assume that general relativity provides an adequate description of gravitational interactions on these cosmological length scales, then the kinematic properties of the Universe

\footnotetext{
${ }^{1}$ Department of Physics, Kansas State University, 116 Cardwell Hall, Manhattan, KS 66506, USA pavlov@phys.ksu.edu,ratra@phys.ksu.edu

${ }^{2}$ Institute of Cosmology and Gravitation, University of Portsmouth, Dennis Sciama Building, Portsmouth, P01 3FX, UK lado.samushia@port.ac.uk
} 
can be derived by solving the Einstein equations

$$
R_{\mu \nu}-\frac{1}{2} g_{\mu \nu} R=8 G \pi T_{\mu \nu}
$$

Here $g_{\mu \nu}$ is the metric tensor, $R_{\mu \nu}$ and $R$ are the Ricci tensor and (curvature) scalar respectively, $T_{\mu \nu}$ is the stress-energy tensor of the Universe's constituents, and $G$ is the Newtonian gravitational constant.

There is good observational evidence that the large-scale radiation and matter distributions are statistically spatially isotropic. The (Copernican) cosmological principle, which is also consistent with current observations, then indicates that the Friedmann-LemaitreRobertson-Walker (FLRW) models provide an adequate description of the spatially homogeneous background cosmological model.

In the FLRW models, the current accelerating cosmological expansion is a consequence of dark energy, the dominant, by far, term in the current cosmological energy budget. The dark energy density could be constant in time (and hence uniform in space) — Einstein's cosmological constant $\Lambda$ (Peebles 1984) — or gradually decreasing in time and thus slowly varying in space (Peebles \& Ratra 1988).

The "standard" model of cosmology is the spatially-flat $\Lambda$ CDM model in which the cosmological constant contributes around $75 \%$ of the current energy budget. Non-relativistic cold dark matter (CDM) is the next largest contributor, at around $20 \%$, with non-relativistic baryons in third place with about $5 \%$. For a review of the standard model see Ratra \& Vogeley (2008) and references therein.

Recent measurements of the anisotropies of the cosmic microwave background (CMB) radiation (e.g., Komatsu et al. 2011; Reichardt et al. 2012), in conjunction with significant observational support for a low density of non-relativistic matter (CDM and baryons together, e.g., Chen \& Ratra 2003), as well as measurements of the position of the baryon acoustic oscillation (BAO) peak in the matter power spectrum (e.g., Percival et al. 2010; Dantas et al. 2011; Carnero et al. 2012; Anderson et al. 2012), provide significant observational support to the spatially-flat $\Lambda$ CDM model. Other data are also not inconsistent with the standard $\Lambda \mathrm{CDM}$ model. These include strong gravitational lensing measurements (e.g., Chae et al. 2004; Lee \& Ng 2007; Biesiada et al. 2010), measurement of Hubble parameter as a function of redshift (e.g., Samushia \& Ratra 2006; Sen \& Scherrer 2008; Pan et al. 2010; Chen \& Ratra 2011), large-scale structure data (e.g., Baldi \& Pettrino 2011; De Boni et al. 2011; Brouzakis et al. 2011; Campanelli et al. 2011), and galaxy cluster gas mass fraction measurements (e.g., Allen et al. 2008; Samushia \& Ratra 2008; Tong \& Noh 2011). For recent reviews of the situation see, e.g., Blanchard (2010), Sapone (2010), and Jimenez (2011). 
While the predictions of the $\Lambda$ CDM model are in reasonable accord with current observations, it is important to bear in mind that dark energy has not been directly detected (and neither has dark matter). Perhaps as a result of this, some feel that it is more reasonable to assume that the left hand side of Einstein's Eq. (1) needs to be modified (instead of postulating a new, dark energy, component of the stress-energy tensor on the right hand side). While such modified gravity models are under active investigation, at present there is no compelling observational reason to prefer any of these over the standard $\Lambda$ CDM cosmological model.

The $\Lambda$ CDM model assumes that dark energy is a cosmological constant with equation of state

$$
p_{\Lambda}=-\rho_{\Lambda}
$$

where $p_{\Lambda}$ and $\rho_{\Lambda}$ are the pressure and energy density of the cosmological constant (fluid). This minimalistic model, despite being in good agreement with most observations available today, has some potential conceptual shortcomings that have prompted research into alternative explanations of the dark energy phenomenon. ${ }^{1}$

To describe possible time-dependence of the dark energy density, it has become popular to consider a more general equation of state parametrization

$$
p_{\omega}=\omega(z) \rho_{\omega}
$$

Here $p_{\omega}$ and $\rho_{\omega}$ are the pressure and energy density of the dark energy fluid with redshift $z$ dependent equation of state parameter $\omega(z)$. The simplest such parametrization is the $\mathrm{XCDM}$ one for which the equation of state parameter is constant and results in accelerated expansion if $\omega(z)=\omega_{X}<-1 / 3$. In this case the dark energy density decreases with time and this allows for the possibility that the fundamental energy density scale for dark energy is set at high energy in the early Universe and the slow decrease of the energy density over the long age of the Universe ensures that the characteristic dark energy density scale now is small (a few meV). This also ensures that the dark energy density remains comparable to the matter energy density over a longer period of time (compared to that for the $\Lambda \mathrm{CDM}$ model).

When $\omega_{X}=-1$ the XCDM parametrization reduces to the consistent (and complete) $\Lambda \mathrm{CDM}$ model. For any other value of $\omega_{X}$ the XCDM parametrization cannot consistently describe spatial inhomogeneities without further assumptions and extension (see, e.g., Ratra 1991; Podariu \& Ratra 2000). Models in which $\omega(z)$ varies in time, $\omega$ CDM models, are also

\footnotetext{
${ }^{1}$ Structure formation in the $\Lambda$ CDM model is governed by the "standard" CDM structure formation model, which might be in some observational difficulty (see, e.g., Peebles \& Ratra 2003; Perivolaropoulos 2010).
} 
unable to consistently describe spatial inhomogeneities without further assumptions and extension.

A physically and observationally viable alternative to the $\Lambda \mathrm{CDM}$ model, that consistently describes a slowly decreasing in time dark energy density, is the $\phi$ CDM model (Peebles \& Ratra 1988; Ratra \& Peebles 1988). This model, in which a dark energy scalar field, $\phi$, slowly roles down its potential, resulting in a slowly decreasing dark energy density, alleviates some of the conceptual problems, mentioned above, associated with the $\Lambda$ CDM model. The slowly rolling scalar field, at a given instant of time, can be approximated by a dark energy fluid with an appropriately negative equation of state parameter.

More specifically, a $\phi \mathrm{CDM}$ model with an inverse-power-law scalar field potential energy density $V(\phi) \propto \phi^{-\alpha}, \alpha>0$, is a prototypical example that has been extensively studied. This model has a non-linear attractor or "tracker" scalar field solution that forces the initially sub-dominant dark energy density to come to dominate over the matter energy density, thus dominating the energy budget of the current Universe, and so resulting in the current accelerated cosmological expansion. In addition to therefore partially alleviating the "coincidence" problem of the $\Lambda \mathrm{CDM}$ model, the $\phi \mathrm{CDM}$ model generates the current tiny dark energy scale of order an meV, measured by the SNeIa, through decrease, via cosmological expansion over the long age of the Universe, of a much larger energy scale.

The $\alpha$ parameter controls the steepness of the scalar field potential, with larger values resulting in a stronger time dependence of the approximate equation of state parameter and $\alpha=0$ corresponds to the $\Lambda$ CDM model limit. $\alpha$ has been constrained using currently available data (see e.g., Chen \& Ratra 2004; Wilson et al. 2006; Chen \& Ratra 2012; Mania $\&$ Ratra 2012, and references therein). The strongest current limits are that $\alpha$ has to be less than $\sim 0.7$ at $2 \sigma$ confidence (Samushia 2009).

In the $\phi \mathrm{CDM}$ model, or in the XCDM or $\omega \mathrm{CDM}$ parameterizations, the background evolution of the (spatially homogeneous) Universe differs from that in the $\Lambda$ CDM case. This affects both the distance-redshift relation as well as the growth rate of large-scale structure. With precise measurements of distance and growth rate over a range of redshifts it will be possible to discriminate between cosmological models. ${ }^{2}$

The BAO signature in the observed large-scale structure of the Universe allows for the measurements of radial and angular distances as functions of redshift (see, e.g., Percival et al.

\footnotetext{
${ }^{2}$ There are many other models under current discussion, besides the $\Lambda$ CDM and $\phi \mathrm{CDM}$ models and $\mathrm{XCDM}$ and $\omega \mathrm{CDM}$ parameterizations we consider here for illustrative purposes. For a sample of the available options see, e.g., Yang et al. (2011), Frolov \& Guo (2011), Nunes et al. (2011), Grande et al. (2011), Saitou \& Nojiri (2011), Silva et al. (2010), Kamenshchik et al. (2011), and Maggiore et al. (2011).
} 
2010; Blake et al. 2011b; Beutler et al. 2011; Anderson et al. 2012). In addition, the redshiftspace distortion signal allows for inferences about the strength of gravitational interactions on very large scales (see, e.g., Percival et al. 2004; Angulo et al. 2007; Guzzo et al. 2008; Blake et al. 2011a; Samushia et al. 2012; Reid et al. 2012). Currently available data sets have been used to measure distances and growth history up to a redshift $z \sim 0.8$ and the next generation of planned space-based galaxy redshift surveys of the whole extragalactic sky are expected to extend these measurements to a redshift $z \sim 2$. Possible candidates for such surveys include the Euclid satellite mission that has been approved by the European Space Agency (Laureijs et al. 2011) and the WFIRST satellite that was ranked high by the recent Decadal Survey (Green et al. 2011). These surveys have been shown to have the potential to measure angular distances, Hubble parameter $H(z)$, and growth rate as functions of redshift to a few percent precision over a wide range of redshifts (Wang et al. 2010; Samushia et al. 2011; Majerotto et al. 2012; Basse et al. 2012). ${ }^{3}$

As mentioned above, an alternative potential explanation of the observed accelerated expansion of the Universe is to replace general relativity by a modified theory of gravity. For example, in the $f(R)$-gravity models the Einstein-Hilbert gravitational action is modified to

$$
S=\frac{1}{16 \pi G} \int d^{4} x \sqrt{-g} f(R),
$$

where the function $f(R)$ of the Ricci curvature $R$ can in general be of any form. In the special case when $f(R)=R$ one recovers the Einstein-Hilbert action which yields the Einstein equations of general relativity, Eq. (1). For every dark energy model it is possible to find a function $f(R)$ that will result in exactly the same expansion history (see, e.g. Sotiriou \& Faraoni 2010; Tsujikawa 2010; Capazziello \& De Laurentis 2011) thus potentially eliminating the need for dark energy. However, nothing prevents the coexistence of modified gravity and dark energy, with both contributing to powering the current accelerated cosmological expansion. It is of significant importance to be able to determine which scenario best describes what is taking place in our Universe.

In this paper we investigate how well anticipated data from the galaxy surveys mentioned above can constrain the time dependence of the dark energy. We will use the Fisher matrix formalism to obtain predictions for the $\phi \mathrm{CDM}$ model and compare these with those made using the (model-dependent) XCDM and $\omega \mathrm{CDM}$ parameterizations of dark energy. We will mostly assume that gravity is well described by general relativity, but will also look at some simple modified gravity cases. We find that the anticipated constraints on the parameter $\alpha$

\footnotetext{
${ }^{3}$ For constraints on cosmological parameters from data from space missions proposed earlier, see Podariu et al. (2001) and references therein.
} 
of the $\phi \mathrm{CDM}$ model are almost an order of magnitude better than the ones that are currently available.

Compared to the recent analysis of Samushia et al. (2011), here we use an updated characterization of planned next-generation space-based galaxy surveys, so our forecasts are a little more realistic. We also consider an additional dark energy parametrization, XCDM, a special case of $\omega$ CDM that was considered by Samushia et al. (2011), as well as the $\phi$ CDM model, forecasting for which has not previously been done.

The paper is organized as follows. In Sec. 2 we briefly describe the observables and their relationship to basic cosmological parameters. In Sec. 3 we describe the models of dark energy that we study. Section 4 outlines the method we use for predicting parameter constraints, with some details given in the Appendix. We present our results in Sec. 5 and conclude in Sec. 6.

\section{Measured power spectrum of galaxies}

The large-scale structure of the Universe, which most likely originated as quantummechanical fluctuations of the scalar field that drove an early epoch of inflation (see, e.g., Fischler et al. 1985), became (electromagnetically) observable at $z \sim 10^{3}$ after the recombination epoch. Dark energy did not play a significant role at this early recombination epoch because of its low mass-density relative to the densities of ordinary and dark matter as well as that of radiation (neutrinos and photons). At $z \sim 5$ galaxy clusters began to form. Initially, in regions where the matter density was a bit higher than the average, space expanded a bit slower than average. Eventually the dark and ordinary matter reached a minimum density and the regions contracted. If an over-dense region was sufficiently large its baryonic matter collapsed into its dark-matter halo. The baryonic matter continued to contract even more due to its ability to lose thermal energy through the emission of electromagnetic radiation. This can not happen with dark matter since it does not emit significant electromagnetic radiation nor does it interact significantly (non-gravitationally) with baryonic matter. As a result the dark matter remained in the form of a spherical halo around the rest of the baryonic part of a galaxy. At $z \sim 2$ the rich clusters of galaxies were formed by gravity, which gathered near-by galaxies together. Also by this time the dark energy's energy density had become relatively large enough to affect the growth of large-scale structure.

Different cosmological models with different sets of parameters can result in the same expansion history and so it impossible to distinguish between such models by using only expansion history measurements. This is one place where measurements of the growth 
history of the large-scale structure of the Universe plays an important role. It is not possible to fix free parameters of two different cosmological models to give exactly the same expansion and growth histories simultaneously. It is therefore vital to observe both histories in order to obtain better constraints on parameters of a cosmological model.

In a cosmological model described by the FLRW metric, and to lowest order in dark matter over-density perturbations, the power-spectrum of observed galaxies is given by (Kaiser 1987)

$$
P_{g}(k, \mu)=P_{m}(k)\left(b \sigma_{8}+f \sigma_{8} \mu^{2}\right)^{2} .
$$

Here subscript $g$ denotes galaxies, $P_{m}$ is the underlying matter power spectrum, $b$ is the bias of galaxies, $f$ is the growth rate, $\mu$ is the cosine of the angle between wave-vector $k$ and the line-of-sight direction, and $\sigma_{8}$ is the overall normalization of the power spectrum $\left(\sigma_{8}\right.$ is the rms energy density perturbation smoothed over spheres of radius $8 h^{-1} \mathrm{Mpc}$, where $h=$ $H_{0} /\left(100 \mathrm{kms}^{-1} \mathrm{Mpc}^{-1}\right)$ and $H_{0}$ is the Hubble constant $)$. Since, for a measured power spectrum of galaxies on a single redshift slice, the bias and growth rate are perfectly degenerate with the overall amplitude, in the equations below we will refer to $b \sigma_{8}$ and $f \sigma_{8}$ simply as $b$ and $f$.

The angular dependence of the power spectrum in Eq. (5) can be used to infer the growth rate factor $f(z)$ which is defined as the logarithmic derivative of the linear growth factor

$$
f(z)=\frac{d \ln G}{d \ln a},
$$

where $a$ is the cosmological scale factor, and the linear growth factor $G(t)=\delta(t) / \delta\left(t_{\text {in }}\right)$ shows by how much the perturbations have grown since some initial time $t_{\mathrm{in}} \cdot{ }^{4}$

The numerical value of the $f(z)$ function depends both on the theory of gravity and on the expansion rate of the Universe. Since the growth rate depends very sensitively on the total amount of non-relativistic matter, it is often parametrized as (see, e.g., Linder 2005, and references therein)

$$
f(z) \approx \Omega_{m}^{\gamma}(z)
$$

where

$$
\Omega_{m}(z)=\frac{\Omega_{m}(1+z)^{3}}{E^{2}(z)}
$$

and

$$
E(z)=H(z) / H_{0}=\sqrt{\Omega_{m}(1+z)^{3}+\Omega_{k}(1+z)^{2}+\Omega_{D E}(z)} .
$$

\footnotetext{
${ }^{4}$ Here we have expanded the energy density $\rho(t, \mathbf{x})$ in terms of a small spatially inhomogeneous fractional perturbation $\delta(t, \mathbf{x})$ about a spatially-homogeneous background $\rho_{b}(t): \rho(t, \mathbf{x})=\rho_{b}(t)[1+\delta(t, \mathbf{x})]$.
} 
Here $H(z)$ is the Hubble parameter and $H_{0}$ is its value at the present epoch (the Hubble constant), $\Omega_{m}$ is the value of the energy density parameter of non-relativistic matter at the present epoch $(z=0), \Omega_{k}$ that of spatial curvature, and $\Omega_{D E}(z)$ is the energy density parameter which describes the evolution of the dark energy density and is different in different dark energy models.

The growth index, $\gamma$, depends on both a model of dark energy as well as a theory of gravity. When general relativity is assumed and the equation of state of dark energy is taken to be of the general form in Eq. (3) then (see, e.g., Linder 2005, and references therein)

$$
\gamma \approx 0.55+0.05[1+\omega(z=1)]
$$

to a few percent accuracy. In the $\Lambda \mathrm{CDM}$ cosmological model $\gamma \approx 0.55$. An observed significant deviation from this value of $\gamma$ will present a serious challenge for the standard cosmological model.

The power spectrum is measured under the assumption of a fiducial cosmological model. If the angular and radial distances in the fiducial model differ from those in the real cosmology, the power spectrum will acquire an additional angular dependence via the Alcock \& Paczyń ski (1979, AP) effect, as discussed in Samushia et al. (2011),

$$
P_{g}(k, \mu)=\frac{1}{f_{\|} f_{\perp}^{2}} P_{m}\left(\frac{k}{f_{\perp} F} \sqrt{F^{2}+\mu^{2}\left(1-F^{2}\right)}\right) \times\left\{b+\frac{\mu^{2} f}{F^{2}+\mu^{2}\left(1-F^{2}\right)}\right\}^{2},
$$

where

$$
\begin{gathered}
f_{\|}(z)=R_{r}(z) / \hat{R}_{r}(z), \\
f_{\perp}(z)=D_{A}(z) / \hat{D}_{A}(z), \\
F=f_{\|} / f_{\perp} .
\end{gathered}
$$

Here $R_{r}=d r / d z$ is the derivative of the radial distance, $D_{A}$ is the angular diameter distance (both defined below), a hat indicates a quantity evaluated in the fiducial cosmological model, and a quantity without a hat is evaluated using the alternative cosmological model. The AP effect is an additional source of anisotropy in the measured power spectrum and allows for the derivation of stronger constraints on cosmological parameters. 


\section{Cosmological models}

In an FLRW model with only non-relativistic matter and dark energy the distances $D_{A}(z)$ and $R_{r}(z)$ are

$$
\begin{gathered}
D_{A}(z)=\frac{1}{h \sqrt{\Omega_{k}}(1+z)} \sinh \left(\sqrt{\Omega_{k}} \int_{0}^{z} \frac{d z^{\prime}}{E\left(z^{\prime}\right)}\right), \\
R_{r}(z)=\frac{1}{h(1+z) E(z)} .
\end{gathered}
$$

Here $E(z)$ is defined in Eq. (9). The functional form of $E(z)$ depends on the model of dark energy.

\section{1. $\Lambda \mathrm{CDM}, \mathrm{XCDM}$ and $\omega \mathrm{CDM}$ parameterizations}

Here we describe the relevant features of the $\Lambda$ CDM model and the dark energy parameterizations we consider.

If the dark energy is taken to be a fluid its equation of state can be written as $p=\omega(z) \rho$. For the $\Lambda$ CDM model the equation of state parameter $\omega(z)=-1$ and the dark energy density is time independent.

In the XCDM parametrization $\omega(z)=\omega_{X}(<-1 / 3)$ is allowed to take any timeindependent value, resulting in a time-dependent dark energy density.

In the $\omega \mathrm{CDM}$ parametrization the time dependence of $\omega(z)$ is parametrized by introducing an additional parameter $\omega_{a}$ through (Chevallier \& Polarski 2001; Linder 2003)

$$
w(z)=w_{0}+w_{a} \frac{z}{1+z} .
$$

The XCDM parametrization is the limit of the $\omega$ CDM parametrization with $\omega_{a}=0$. In the $\omega \mathrm{CDM}$ parametrization the function $\Omega_{D E}(z)$ that describes the time evolution of the dark energy density is

$$
\Omega_{D E}(z)=\left(1-\Omega_{m}-\Omega_{k}\right)(1+z)^{3\left(1+w_{0}+w_{a}\right)} \exp \left(-3 w_{a} \frac{z}{1+z}\right)
$$

and the corresponding expression for the XCDM case can be derived by setting $\omega_{a}=0$ here. 


\section{2. $\phi$ CDM model}

In the $\phi \mathrm{CDM}$ model the energy density of the background, spatially homogeneous, scalar field $\phi$ can be found by solving the set of simultaneous ordinary differential equations of motion,

$$
\begin{gathered}
\ddot{\phi}+3 \frac{\dot{a}}{a} \dot{\phi}+V^{\prime}(\phi)=0, \\
\left(\frac{\dot{a}}{a}\right)^{2}=\frac{8 \pi G}{3}\left(\rho+\rho_{\phi}\right)-\frac{k}{a^{2}}, \\
\rho_{\phi}=\frac{1}{16 \pi G}\left(\frac{1}{2} \dot{\phi}^{2}+V(\phi)\right) .
\end{gathered}
$$

Here an over-dot denotes a derivative with respect to time, a prime denotes one with respect to $\phi, V(\phi)$ is the potential energy density of the scalar field, $\rho_{\phi}$ is the energy density of the scalar field, and $\rho$ that of the other constituents of the Universe.

Following Peebles \& Ratra (1988) we consider a scalar field with inverse-power-law potential energy density

$$
V(\phi)=\frac{\kappa}{2 G} \phi^{-\alpha}
$$

Here $\alpha$ is a positive parameter of the model to be determined experimentally and $\kappa$ is a positive constant. This choice of potential has the interesting property that the scalar field solution is an attractor with an energy density that slowly comes to dominate over the energy density of the non-relativistic matter (in the matter dominated epoch) and causes the cosmological expansion to accelerate. The function $\Omega_{D E}(z)$ in the case of $\phi \mathrm{CDM}$ is

$$
\Omega_{D E}(z)=\frac{1}{12}\left(\dot{\phi}^{2}+\frac{\kappa}{G} \phi^{-\alpha}\right)
$$

\section{Fisher matrix formalism}

The precision of the galaxy power spectrum measured in redshift bins depends on the cosmological model, the volume of the survey, and the distribution of galaxies within the observed volume. See App. A for a summary of how to estimate the precision of measurements from survey parameters.

We assume that the power spectrum $P\left(k_{i}\right)^{\text {meas }}$ has been measured in $N$ wave-number $k_{i}$ bins $(i=1 \ldots N)$ and each measurement has a Gaussian uncertainty $\sigma_{i}$. From these measurements a likelihood function

$$
\mathcal{L} \propto \exp \left(-\frac{1}{2} \chi^{2}\right)
$$


can be constructed where

$$
\chi^{2}=\sum_{i=1}^{N} \frac{\left(P_{i}^{\text {meas }}-P_{i}(\mathbf{p})\right)^{2}}{\sigma_{i}^{2}} .
$$

Here $\mathbf{p}$ are the set of cosmological parameters on which the power spectrum depends.

The likelihood function in Eq. (24) can be transformed into the likelihood of theoretical parameters $\mathbf{p}$ by Taylor expanding it around the maximum and keeping terms of only second order in $\delta \mathbf{p}$ as $\chi^{2}(\delta p)=F_{j k} \delta p^{j} \delta p^{k}$, where $F_{j k}$ is the Fisher matrix ${ }^{5}$ of the parameter set $\mathbf{p}$ given by second derivatives of the likelihood function through

$$
F_{j k}=-\left\langle\frac{\partial^{2} \ln \mathcal{L}}{\partial p^{j} \partial p^{k}}\right\rangle
$$

The Fisher matrix predictions are exact in the limit where initial measurements as well as derived parameters are realizations of a Gaussian random variable. This would be the case if the $P_{i}^{\text {meas }}$ were perfectly Gaussian and the $P_{i}(\mathbf{p})$ were linear functions of $\mathbf{p}$, which would make the second order Taylor expansion of the likelihood around its best fit value exact. In reality, because of initial non-Gaussian contributions and nonlinear effects, the predictions of Fisher matrix analysis will be different (more optimistic) from what is achievable in practice. These differences are larger for strongly non-linear models and for the phase spaces in which the likelihood is non-negligible at some physical boundary $(\alpha=0$ in case of $\phi \mathrm{CDM})$. A more realistic approach, that requires significantly more computational time and power, is to generate a large amount of mock data and perform a full Monte-Carlo Markov Chain (MCMC) analysis (see, e.g., Perotto et al. 2006; Martinelli et al. 2011, where the authors find significant differences compared to the results of the Fisher matrix analysis).

We assume that the full-sky space-based survey will observe $\mathrm{H} \alpha$-emitter galaxies over $15000 \operatorname{deg}^{2}$ of the sky. For the density and bias of observed galaxies we use predictions from Orsi et al. (2010) and Geach et al. (2010) respectively. We further assume that about half of the galaxies will be detected with a reliable redshift. These numbers roughly mirror what proposed space missions, such as the ESA Euclid satellite and the NASA WFIRST mission, are anticipated to achieve. For the fiducial cosmology we use a spatially-flat $\Lambda$ CDM model with $\Omega_{m}=0.25$, the baryonic matter density parameter $\Omega_{b}=0.05, \sigma_{8}=0.8$, and the primordial density perturbation power spectral index $n_{s}=1.0$, for convenience we summarize all the parameters of the fiducial model in Table 1.

${ }^{5}$ For a review of the Fisher matrix formalism as applied to cosmological forecasting, see Albrecht et al. (2009). 
Table 1: Values of the parameters of the fiducial $\Lambda$ CDM model and the survey.

\begin{tabular}{|c|c|c|c|c|c|c|c|c|}
\hline$\Omega_{m}$ & $\Omega_{b}$ & $\Omega_{k}$ & $\mathrm{~h}$ & $\sigma_{8}$ & $n_{s}$ & Efficiency & Redshift span & Covered sky area in deg \\
\hline 0.25 & 0.05 & 0.0 & 0.7 & 0.8 & 1.0 & 0.45 & $0.55 \leq z \leq 2.05$ & 15000 \\
\hline
\end{tabular}

We further assume that the shape of the power spectrum is known perfectly (for example from the results of the Planck satellite) and ignore derivatives of the real-space power spectrum with respect to cosmological parameters.

We predict the precision of the measured galaxy power spectrum and then transform it into correlated error bars on the derived cosmological parameters. At first we make predictions for the basic quantities $b$ and $f$ in the XCDM and $\omega \mathrm{CDM}$ parameterizations and in the $\phi \mathrm{CDM}$ model. Then it allows us to predict constraints on deviations from general relativity and see how these results change with changing assumptions about dark energy. Finally, we forecast constraints on the basic cosmological parameters of dark energy models.

For the XCDM parametrization these basic cosmological parameters are $p_{\mathrm{XCDM}}=$ $\left(f, b, h, \Omega_{m}, \Omega_{k}, w_{X}\right)$. The $\omega \mathrm{CDM}$ parametrization has one extra parameter describing the time evolution of the dark energy equation of state parameter, $p_{\omega \mathrm{CDM}}=\left(f, b, h, \Omega_{m}, \Omega_{k}, w_{0}, w_{a}\right)$. For the $\phi \mathrm{CDM}$ model the time dependence of the dark energy density depends only on one parameter $\alpha$ so we have $p_{\phi \mathrm{CDM}}=\left(f, b, h, \Omega_{m}, \Omega_{k}, \alpha\right)$. In order to derive constraints on the parameters of the considered cosmological models while altering assumptions about the correctness of general relativity, we transform Fisher matrices of each model from the parameter set described above to the following parameter set (that now includes $\gamma$ that parametrizes the growth rate) $p_{\text {model }}=(\gamma$, model $)$, where by model we mean all the parameters of a particular model, for example, for $\omega \mathrm{CDM}$ model $=p_{\omega \mathrm{CDM}}=\left(f, b, h, \Omega_{m}, \Omega_{k}, w_{0}, w_{a}\right)$.

\section{Results}

\subsection{Constraints on growth rate}

Figure 1 shows predictions for the measurement of growth rate assuming different dark energy models. We find that in the most general case, when no assumption is made about the nature of dark energy, the growth rate can be constrained to a precision of better then $2 \%$ over a wide range of redshifts. This is in good agreement with previous similar studies (see, e.g., Fig. 1 of Samushia et al. 2011). When we specify a dark energy model the constraints on growth rate improve by about a factor of two. There is very little difference between the results derived for different dark energy models: the precision is almost insensitive to the 
assumed model. Also, one can notice that the curves for the XCDM parametrization and for the $\phi \mathrm{CDM}$ model are almost identical. The likely explanation of this effect is that for a fixed redshift bin the $\phi \mathrm{CDM}$ model is well described by the XCDM parametrization with the value of the parameter $\omega_{X}=p_{\phi} / \rho_{\phi}$, where the values of the scalar field pressure $p_{\phi}$ and energy density $\rho_{\phi}$ are evaluated at that redshift bin.

The measurements of growth rate can be remapped into constraints on parameters describing the deviation from general relativity. Figure 2 shows correlated constraints between the current re-normalized Hubble constant $h$ and the $\gamma$ parameter that describes the growth of structure. The $\phi \mathrm{CDM}$ model constraints on both $h$ and $\gamma$ are tighter than those for the $\mathrm{XCDM}$ or $\omega \mathrm{CDM}$ parameterizations. As expected, the most restrictive $\Lambda$ CDM model results in the tightest constraints. In Table 2 we show the deviations of the parameter $\gamma$ from its fiducial value for various dark energy models.

\subsection{Constraints on dark energy model parameters}

We use measurements of growth and distance to constrain parameters of the dark energy models.

Figure 3 shows constraints on parameters of the $\omega$ CDM parameterization (these should be compared to Figs. 4a and 5a of Samushia et al. 2011). When no assumptions are made about the nature of gravity the constraints on $\omega_{0}$ and $\omega_{a}$ are very weak and degenerate. When we assume general relativity the constraints tighten significantly, resulting in $\sim 10 \%$ accuracy in the measurement of $\omega_{0}$ and $\sim 25 \%$ accuracy in the measurement of $\omega_{a}$.

The upper panel of Fig. 4 shows constraints on the parameters $\omega_{X}$ and $\Omega_{m}$ of the XCDM parametrization. Similar to the previous case, the constraints tighten significantly when we assume general relativity as the model of gravity. About a $2 \%$ measurement of $\omega_{X}$ and a $5 \%$ measurement of $\Omega_{m}$ are possible in this case. The lower panel of Fig. 4 show the related constraints on $\Omega_{k}$ and $\Omega_{m}$ for the XCDM parametrization. The constraints are similar to, but somewhat tighter than, those for the $\omega$ CDM parametrization. This is because the XCDM parametrization has one less parameter than the $\omega$ CDM parametrization. Spatial curvature can be constrained to about $15 \%$ precision in this case.

Figures 5 and 6 show constraints on parameters of the $\phi$ CDM model. In the most general case, when no assumption is made about the nature of gravity, the constraints are weak and the parameters $\alpha$ and $\Omega_{m}$ are strongly correlated, with larger values of $\alpha$ requiring larger values of $\Omega_{m}$. When general relativity is assumed, the constraints become much stronger and parameter $\alpha$ can be constrained to be less than 0.1 at the $1-\sigma$ confidence level. This is 
significantly better than any constraint available at the moment.

Figure 7 shows constraints on the parameters of the $\Lambda$ CDM model. From the clustering data alone the spatial curvature can be constrained with almost $1 \%$ precision, largely because this model has the least number of free parameters.

The exact numerical values for the forecast error bars and likelihood contours should be taken with caution and not be interpreted as predictions for the performance of any specific survey (such as Euclid or WFIRST). Our main objective in this work was first to investigate how the modified gravity constraints change with different models of dark energy and second to demonstrate the improvement in $\phi \mathrm{CDM}$ model constraints achievable with future galaxy surveys. Because of this we were able to simplify our method by adopting a Fisher matrix formalism instead of a full MCMC approach and also use a simplified description of the survey baseline. For more realistic predictions of Euclid performance, see, e.g., Laureijs et al. (2011); Samushia et al. (2011); Majerotto et al. (2012).

Table 2: Predicted deviations of parameter $\gamma$ from its fiducial value, at one standard deviation confidence level, for different assumptions about dark energy.

\begin{tabular}{|c|c|c|}
\hline DE model & Fiducial $\gamma$ & deviation \\
\hline$\omega \mathrm{CDM}$ & 0.55 & 0.035 \\
$\phi \mathrm{CDM}$ & 0.55 & 0.023 \\
$\mathrm{XCDM}$ & 0.55 & 0.035 \\
$\Lambda \mathrm{CDM}$ & 0.55 & 0.016 \\
\hline
\end{tabular}

\section{Conclusion}

We have forecast the precision at which planned near-future space-based spectroscopic galaxy surveys should be able to constrain the time dependence of dark energy density. For the first time, we have used a consistent physical model of time-evolving dark energy, $\phi \mathrm{CDM}$, in which a minimally-coupled scalar field slowly rolls down its self-interaction potential energy density. We have shown that if general relativity is assumed, the deviation of the parameter $\alpha$ of the $\phi \mathrm{CDM}$ model can be constrained to better than 0.05 ; this is almost an order of magnitude better than the best currently available result.

The constraints on basic cosmological parameters, such as the relative energy densities of non-relativistic matter and spatial curvature, depend on the adopted dark energy model. We have shown that in the $\phi \mathrm{CDM}$ model the expected constraints are more restrictive than those derived using the XCDM or $\omega \mathrm{CDM}$ parameterizations. This is due to the fact that 
the $\phi \mathrm{CDM}$ model has fewer parameters. Also, the XCDM and $\omega \mathrm{CDM}$ parameterizations assign equal weight to all possible values of $\omega$, while in the $\phi \mathrm{CDM}$ model there is an implicit theoretical prior on which equation of state parameter values are more likely, based on how easy it is to produce such a value within the model.

Since the observational consequences of dark energy and modified gravity are partially degenerate, constraints on modified gravity parameters will depend on the assumptions made about dark energy. The constraints on $\gamma$ are most restrictive in the $\Lambda$ CDM model. For the $\phi \mathrm{CDM}$ model the constraints on $\gamma$ are about a third tighter than those for the $\omega \mathrm{CDM}$ and $\mathrm{XCDM}$ parameterizations.

These results are very encouraging: data from an experiment of the type we have modeled will be able to provide very good, and probably revolutionary, constraints on the time evolution of dark energy.

\section{Acknowledgments}

This work was supported by DOE grant DEFG030-99EP41093 and NSF grant AST1109275. LS is grateful for support from European Research Council, SNSF SCOPES grant \# 128040, and GNSF grant ST08/4-442.

\section{A. Appendix}

In this Appendix we summarize how to estimate the precision of measurements from the survey parameters.

The Fisher matrix coefficients are given by

$$
F_{i j}=\frac{1}{2} \int_{k_{\min }}^{k_{\max }}\left(\frac{\partial \ln P}{\partial p^{i}}\right)\left(\frac{\partial \ln P}{\partial p^{j}}\right) V_{\text {eff }}(k, \mu) \frac{d^{3} k}{(2 \pi)^{3}},
$$

where the effective volume is

$$
V_{\text {eff }}=V_{0} \frac{n P(k, \mu)}{1+n P(k, \mu)},
$$

and $V_{0}$ is the total survey volume and $n$ is the number density. Also, following Tegmark (1997), we multiply the integrand in Eq. (A1) by a Gaussian factor $\exp \left(-k^{2} \sigma_{z} \frac{d r(z)}{d z}\right)$, where $r(z)$ is the co-moving distance, in order to account for the errors in distance induced by the errors of redshift measurements, $\sigma_{z}=0.001$. We model the theoretical power spectrum using an analytic approximation of Eisenstein \& $\mathrm{Hu}(1998)$. We integrate in $k$ from $k_{\min }=0$ to 
$k_{\max }$, where the $k_{\max }$ values depend on redshift and are chosen in such a way that the small scales that are dominated by non-linear effects are excluded. The range of scales that will be fitted to the future surveys will depend on how well the theoretical templates are able to describe small-scale clustering and is difficult to predict. The $k_{\max }$ values along with the expected bias and number density of galaxies are listed in Table 3.

In order to derive the Fisher matrix of a specific cosmological model we have to go from our initial parameter space to the parameter space of the cosmological model whose Fisher matrix we want. The transformation formula for the Fisher matrix is given by (see, e.g., Albrecht et al. 2009, for a review)

$$
F_{l m}^{\prime}=\frac{\partial p_{i}}{\partial p_{l}^{\prime}} \frac{\partial p_{j}}{\partial p_{m}^{\prime}} F_{i j}
$$

where the primes denote the "new" Fisher matrix and parameters.

We now list the derivatives of the transformation coefficients of the $\phi \mathrm{CDM}$ model in the limit $\alpha \longrightarrow 0$ and $\Omega_{k} \longrightarrow 0$ (which corresponds to the fiducial spatially-flat $\Lambda$ CDM model). The transformation coefficients relating $f_{\|}(z)$ and the parameters $\left(h, \Omega_{m}, \Omega_{k}, \alpha\right)$ are

$$
\begin{gathered}
\frac{\partial f_{\|}(z)}{\partial h}=-\frac{1}{h} \\
\frac{\partial f_{\|}(z)}{\partial \Omega_{m}}=\frac{1}{2 E(z)^{2}}\left[1-(1+z)^{3}\right] \\
\frac{\partial f_{\|}(z)}{\partial \Omega_{k}}=\frac{1}{2 E(z)^{2}}\left[1-(1+z)^{2}\right], \\
\frac{\partial f_{\|}(z)}{\partial \alpha}=-\frac{\left(1-\Omega_{m}\right)}{8 E(z)^{2}} .
\end{gathered}
$$

For the other transformation coefficients, it is convenient to introduce the integral

$$
D(z)=\int_{0}^{z} \frac{d z^{\prime}}{E\left(z^{\prime}\right)}
$$

Then the transformation coefficients between $f_{\perp}(z)$ and the parameters $\left(h, \Omega_{m}, \Omega_{k}, \alpha\right)$ are

$$
\begin{gathered}
\frac{\partial f_{\perp}(z)}{\partial h}=-\frac{1}{h}, \\
\frac{\partial f_{\perp}(z)}{\partial \Omega_{m}}=\frac{1}{2 D(z)} \int_{0}^{z} \frac{d z^{\prime}}{E\left(z^{\prime}\right)^{3}}\left[1-\left(1+z^{\prime}\right)^{3}\right], \\
\frac{\partial f_{\perp}(z)}{\partial \Omega_{k}}=\frac{D(z)^{2}}{6}+\frac{1}{2 D(z)} \int_{0}^{z} \frac{d z^{\prime}}{E\left(z^{\prime}\right)^{3}}\left[1-\left(1+z^{\prime}\right)^{2}\right],
\end{gathered}
$$




$$
\frac{\partial f_{\perp}(z)}{\partial \alpha}=-\frac{\left(1-\Omega_{m}\right)}{8 D(z)} \int_{0}^{z} \frac{d z^{\prime}}{E\left(z^{\prime}\right)^{3}}
$$

Finally, the transformation coefficients between the growth factor $f(z)$ and the parameters $\left(\gamma, h, \Omega_{m}, \Omega_{k}, \alpha\right)$ are

$$
\begin{gathered}
\frac{\partial f(z)}{\partial \gamma}=\frac{f(z)}{\gamma} \ln f(z) \\
\frac{\partial f(z)}{\partial \Omega_{m}}=\frac{\gamma f(z)}{\Omega_{m} E(z)^{2}}\left\{E(z)^{2}-\Omega_{m}\left[(1+z)^{3}-1\right]\right\} \\
\frac{\partial f(z)}{\partial \Omega_{k}}=-\frac{\gamma f(z)}{E(z)^{2}}\left[(1+z)^{2}-1\right] \\
\frac{\partial f(z)}{\partial \alpha}=-\frac{\gamma f(z)}{4 E(z)^{2}}\left[1-\Omega_{m}\right] .
\end{gathered}
$$

Table 3: Values of the $k_{\max }$, bias $b(z)$ from Orsi et al. (2010), and the number densities $n(z)$ taken from Geach et al. (2010).

\begin{tabular}{|c|c|c|c|}
\hline$z$ & $k_{\max }$ & $b(z)$ & $n(z)$ \\
\hline 0.55 & 0.144 & 1.0423 & 3220 \\
\hline 0.65 & 0.153 & 1.0668 & 3821 \\
\hline 0.75 & 0.163 & 1.1084 & 4364 \\
\hline 0.85 & 0.174 & 1.1145 & 4835 \\
\hline 0.95 & 0.185 & 1.1107 & 5255 \\
\hline 1.05 & 0.197 & 1.1652 & 5631 \\
\hline 1.15 & 0.2 & 1.2262 & 5972 \\
\hline 1.25 & 0.2 & 1.2769 & 6290 \\
\hline 1.35 & 0.2 & 1.2960 & 6054 \\
\hline 1.45 & 0.2 & 1.3159 & 4985 \\
\hline 1.55 & 0.2 & 1.4416 & 4119 \\
\hline 1.65 & 0.2 & 1.4915 & 3343 \\
\hline 1.75 & 0.2 & 1.4873 & 2666 \\
\hline 1.85 & 0.2 & 1.5332 & 2090 \\
\hline 1.95 & 0.2 & 1.5705 & 1613 \\
\hline 2.05 & 0.2 & 1.6277 & 1224 \\
\hline
\end{tabular}

\section{REFERENCES}

Albrecht, A., Amendola, L., Bernstein, G., et al. 2009, arXiv:0901.0721 
Alcock, C., \& Paczyń ski, B. 1979, Nature, 281, 358

Allen, S. W., Rapetti, D. A., Schmidt, R. W., et al. 2008, MNRAS, 383, 879

Anderson, L., Aubourg, E., Bailey, S., et al. 2012, arXiv:1203.6594

Angulo, R. E., Baugh, C. M., \& Lacey, C. G. 2007, MNRAS, 387, 921

Baldi, M., \& Pettrino, V. 2011, MNRAS, 412, L1

Barreira, A., \& Avelino, P. P. 2011, Phys. Rev. D, 84, 083521

Basse, T., Eggers Bjaelde, O., Hannestad, S., \& Wong, Y. Y. Y. 2012, arXiv:1205.0548

Beutler, F., Blake, C., Colless, M., et al. 2011, MNRAS, 416, 3017

Biesiada, M., Piórkowska, A., \& Malec, B. 2010, MNRAS, 406, 1055

Blake, C., Brough, S., Colless, M., et al. 2011a, MNRAS, 415, 2876

Blake, C., Kazin, E. A., Beulter, F., et al. 2011b, MNRAS, 418, 1707

Blanchard, A. 2010, A\&AR, 18, 595

Brouzakis, N., Pettorino, V., Tetradis, N.,\& Wetterich, C. 2011, J. Cosmol. Astropart. Phys., JCAP03(2011)049

Campanelli, L., Fogli, G. L., Kahniashvili, T., Marrone, A., \& Ratra, B. 2011, arXiv:1110.2310

Capazziello, S., \& De Laurentis, M. 2011, Phys. Rept., 509, 167

Carnero, A. et al. 2012, MNRAS, 419, 1689

Chae, K.-H., et al. 2004, ApJ, 607, L71

Chen, G., \& Ratra, B. 2003, PASP, 115, 1143

Chen, G., \& Ratra, B. 2004, ApJ, 612, L1

Chen, Y., \& Ratra, B. 2011, Phys. Lett. B, 703, 406

Chen, Y., \& Ratra, B. 2012, A\&A, 543, A104

Chevallier, M., \& Polarski, D. 2001, Int. J. Mod. Phys. D, 10, 213

Conley, A., et al. 2011, ApJS, 192, 1 
Dantas, M. A., et al. 2011, Phys Lett. B, 699, 239

De Boni, C., et al. 2011, MNRAS, 415, 2758

Eisenstein, D. J., \& Hu, W. 1998, ApJ, 496, 605

Fischler, W., Ratra, B., \& Susskind, L. 1985, Nucl. Phys. B, 259, 730

Frolov, A. V., \& Guo, J.-Q. 2011, arXiv:1101.4995 [astro-ph.CO]

Geach, J. E., et al. 2010, MNRAS, 402, 1330

Grande, J., et al. 2011, J. Cosmology Astropart. Phys., 1108, 007

Green, J, et al. 2011, arXiv:1108.1374 [astro-ph.IM]

Guzzo, L., Pierleoni, M., Meneux, B., et al. 2008, Nature., 451, 541

Jimenez, R. 2011, Fortschr. Phys., 59, 602

Kaiser, N. 1987, MNRAS, 227, 1

Kamenshchik, A. Y., Tronconi, A., \& Venturi, G. 2011, Phys. Lett. B, 702, 191

Komatsu, E., et al. 2011, ApJS, 192, 18

Laureijs, R., et al. 2011, arXiv:1110.3193 [astro-ph.CO])

Lee, S., \& Ng, K.-W. 2007, Phys. Rev. D, 76, 043518

Li, X.-D., et al. 2011, J. Cosmology Astropart. Phys., 1107, 011

Linder, E. V. 2003, Phys. Rev. Lett., 90, 091301

Linder, E. V. 2005, Phys. Rev. D, 72, 043529

Maggiore, M., et al. 2011, Phys. Lett. B, 704, 102

Majerotto, E., Guzzo, L., Samushia, L., et al. 2012, MNRAS, 424, 1392, arXiv:1205.6215

Mania, D., \& Ratra, B. 2012, Phys. Lett. B, 715, 9

Martinelli, M., et al. 2011, Phys. Rev. D, 83, 023012

Nunes, N. J., Schrempp, L., \& Wetterich, C. 2011, Phys. Rev. D, 83, 083523

Orsi, A., et al. 2010, MNRAS, 405, 1006 
Pan, N., et al. 2010, Class. Quantum Grav., 27, 155015

Peebles, P. J. E. 1984, ApJ, 284, 439

Peebles, P. J. E., \& Ratra, B. 1988, ApJ, 325, L17

Peebles, P. J. E., \& Ratra, B. 2003, Rev. Mod. Phys., 75, 559

Percival, W. J., et al. 2004, MNRAS, 385, L78

Percival, W. J., et al. 2010, MNRAS, 401, 2148

Perivolaropoulos, L. 2010, J. Phys. Conf. Ser., 222, 012024

Perotto, L., et al. 2006, J. Cosmology Astropart. Phys., 0610, 013

Podariu, S., Nugent, P., \& Ratra, B. 2001, ApJ, 553, 39

Podariu, S., \& Ratra, B. 2000, ApJ, 532, 109

Ratra, B. 1991, Phys. Rev. D, 43, 3802

Ratra, B., \& Peebles, P. J. E. 1988, Phys. Rev. D, 37, 3406

Ratra, B., \& Vogeley, M. S. 2008, PASP, 120, 235

Reichardt, C. L., et al. 2012, ApJ, 749, L9

Reid, B. A., et al. 2012, arXiv:1203.6641

Saitou, R., \& Nojiri, S. 2011, Eur. Phys. J. C, 71, 1712

Samushia, L. 2009, PhD thesis, Kansas State University arXiv:0908.4597

Samushia, L. et al. 2011, MNRAS, 410, 1993

Samushia, L., Percival, W. J., \& Raccanelli, A. 2012, MNRAS, 420, 2102

Samushia, L., \& Ratra, B. 2006, ApJ, 650, L5

Samushia, L., \& Ratra, B. 2008, ApJ, 680, L1

Sapone, D. 2010, Int. J. Mod. Phys. A, 25, 5253

Sen, A. A., \& Scherrer, R. J. 2008, Phys. Lett. B, 659, 457

Silva, R., et al. 2012, A\&A, 537, A11 
Sotiriou, T. P., \& Faraoni, V. 2010, Rev. Mod. Phys., 82, 451

Suzuki, N., et al. 2012, ApJ, 746, 85

Tegmark, M. 1997, Phys. Rev. Lett., 79, 3806

Tong, M., \& Noh, H. 2011, Eur. Phys. J. C, 71, 1586

Tsujikawa, S. 2010, Lectures on Cosmology Accelerated Expansion of the Universe by Georg Wolschin (Lecture Notes in Physics, Vol. 800; Berlin: Springer), 99

Wang, Y., et al. 2010, MNRAS, 409, 737

Wilson, K. M., Chen, G., \& Ratra, B. 2006, Mod. Phys. Lett. A, 21, 2197

Yang, R.-J., Zhu, Z.-H., \& Wu, F. 2011, Int. J. Mod. Phys. A, 26, 317 


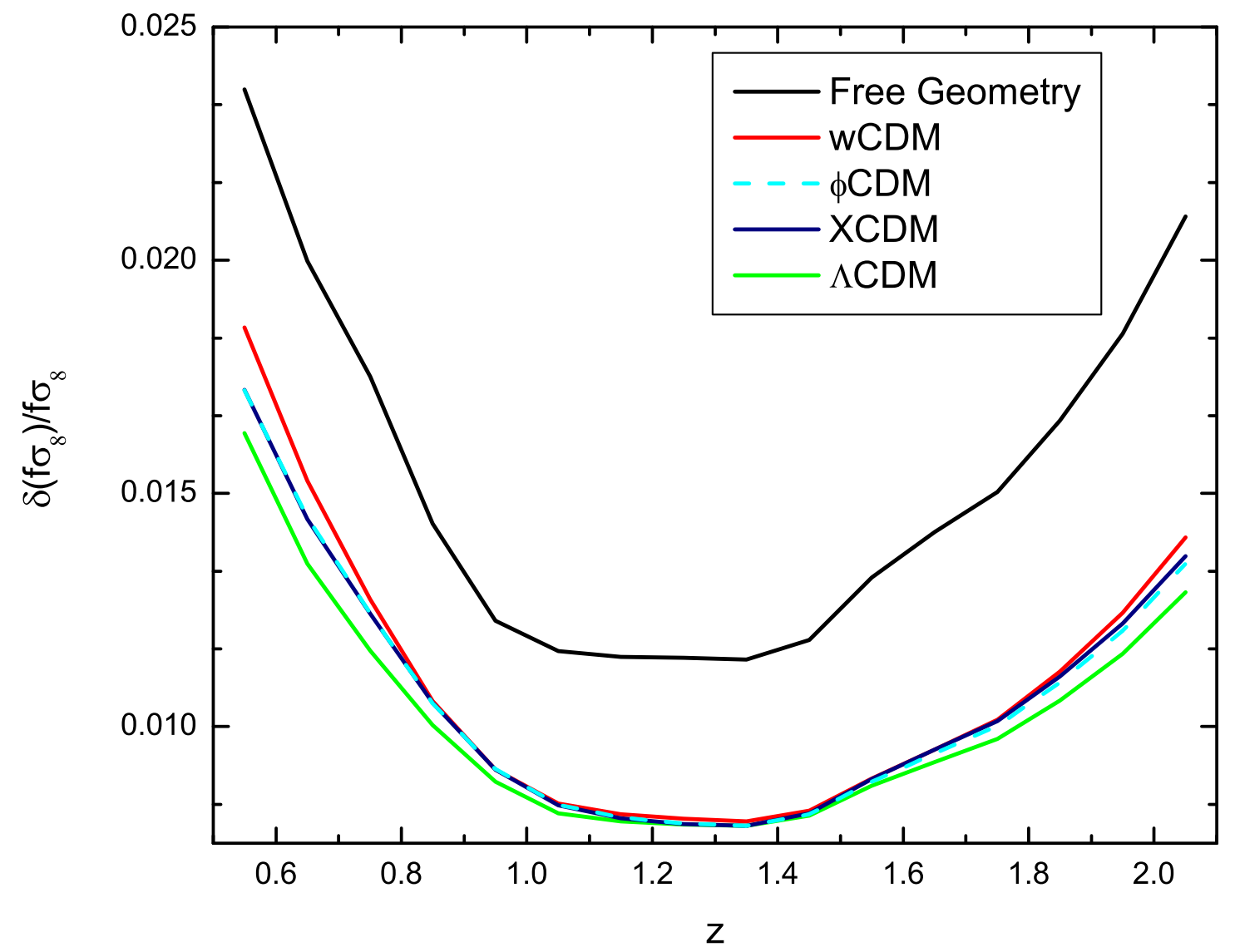

Fig. 1. - Predicted relative error on the measurements of growth rate as a function of redshift $z$ in redshift bins of $\Delta z=0.1$ for different models of dark energy. The upper solid black line shows predictions for the case when no assumption is made about the nature of dark energy. 


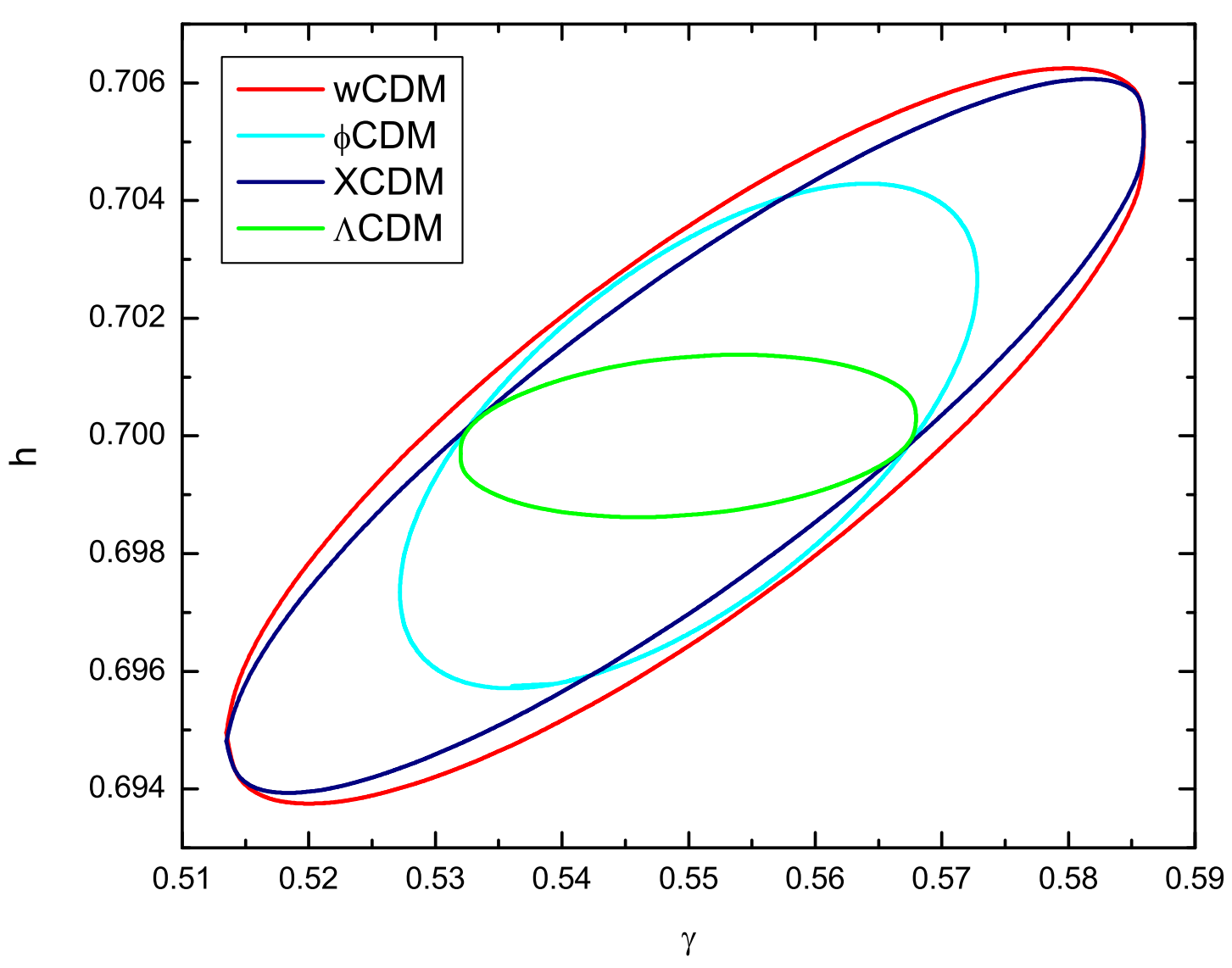

Fig. 2.- Predicted one standard deviation confidence level contour constraints on the current renormalized Hubble constant $h$ and the parameter $\gamma$ that describes deviations from general relativity for different dark energy models. 

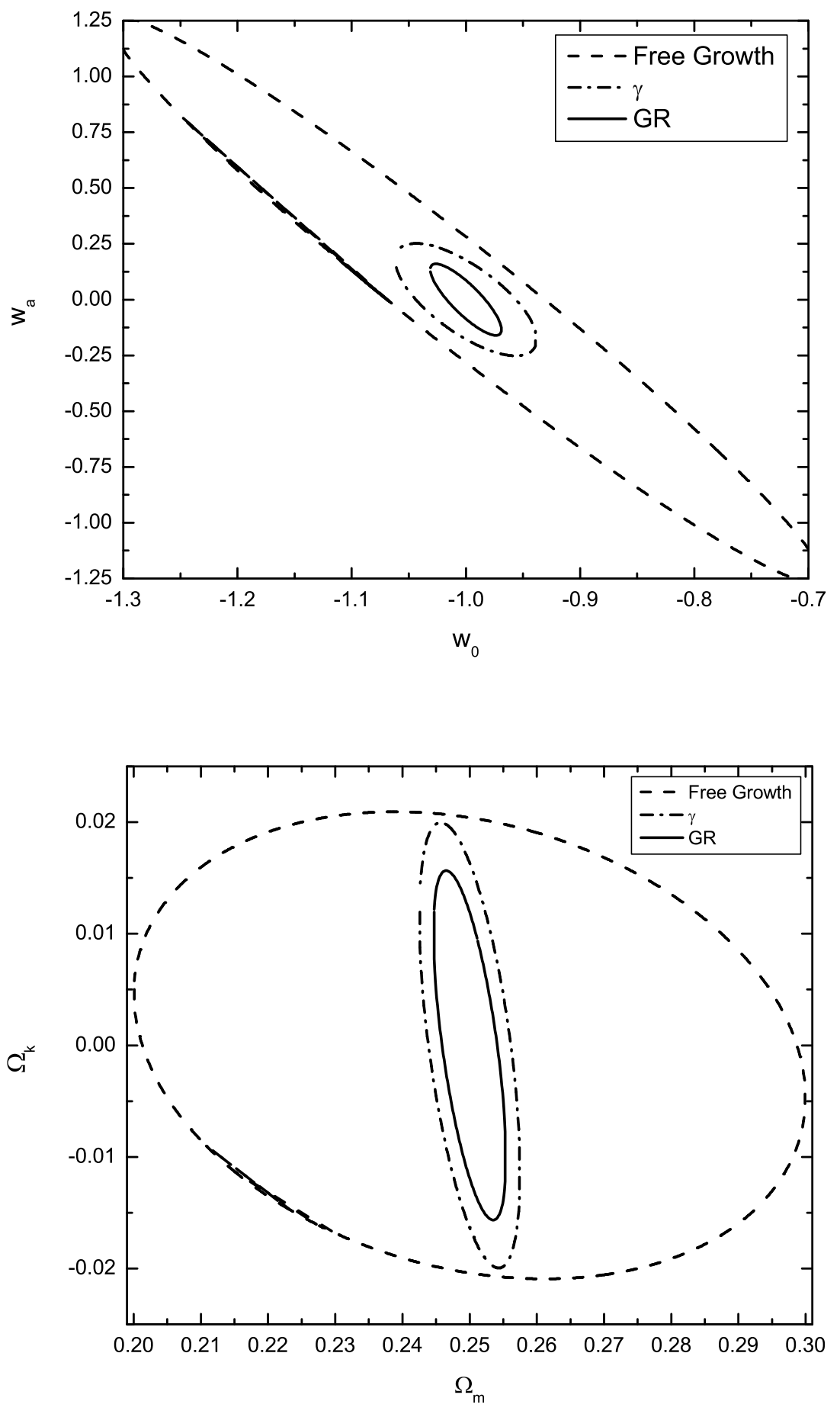

Fig. 3.- Upper panel shows one standard deviation confidence level contours constraints on parameters $\omega_{a}$ and $\omega_{0}$ of the $\omega \mathrm{CDM}$ parametrization, while lower panel shows these for parameters $\Omega_{k}$ and $\Omega_{m}$. 

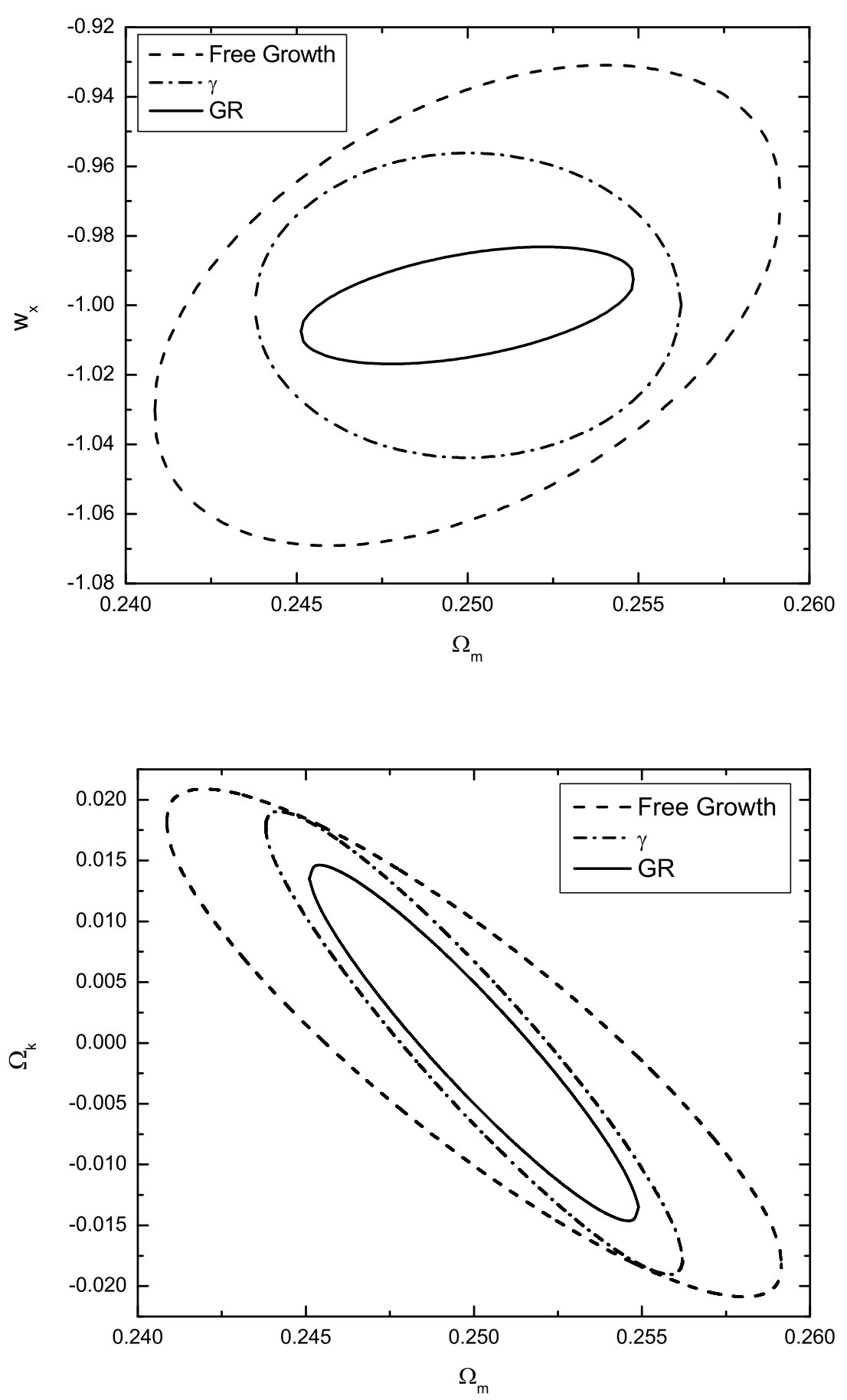

Fig. 4.- One standard deviation confidence level contour constraints on parameters of the XCDM parametrization. 

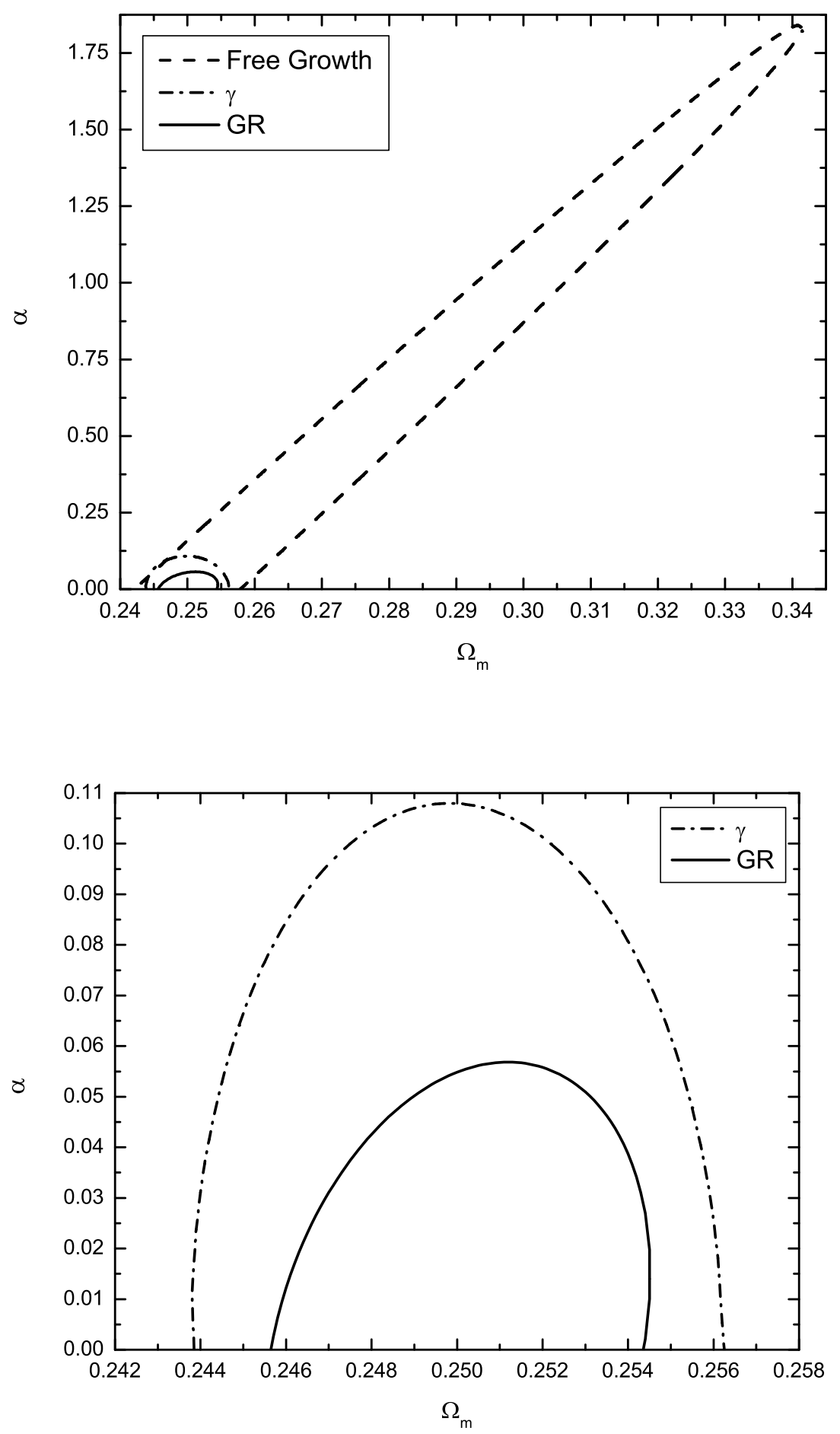

Fig. 5.- One standard deviation confidence level contour constraints on parameters $\alpha$ and $\Omega_{m}$ of the $\phi \mathrm{CDM}$ model. Lower panel shows a magnification of the tightest two contours in the lower left corner of the upper panel. 

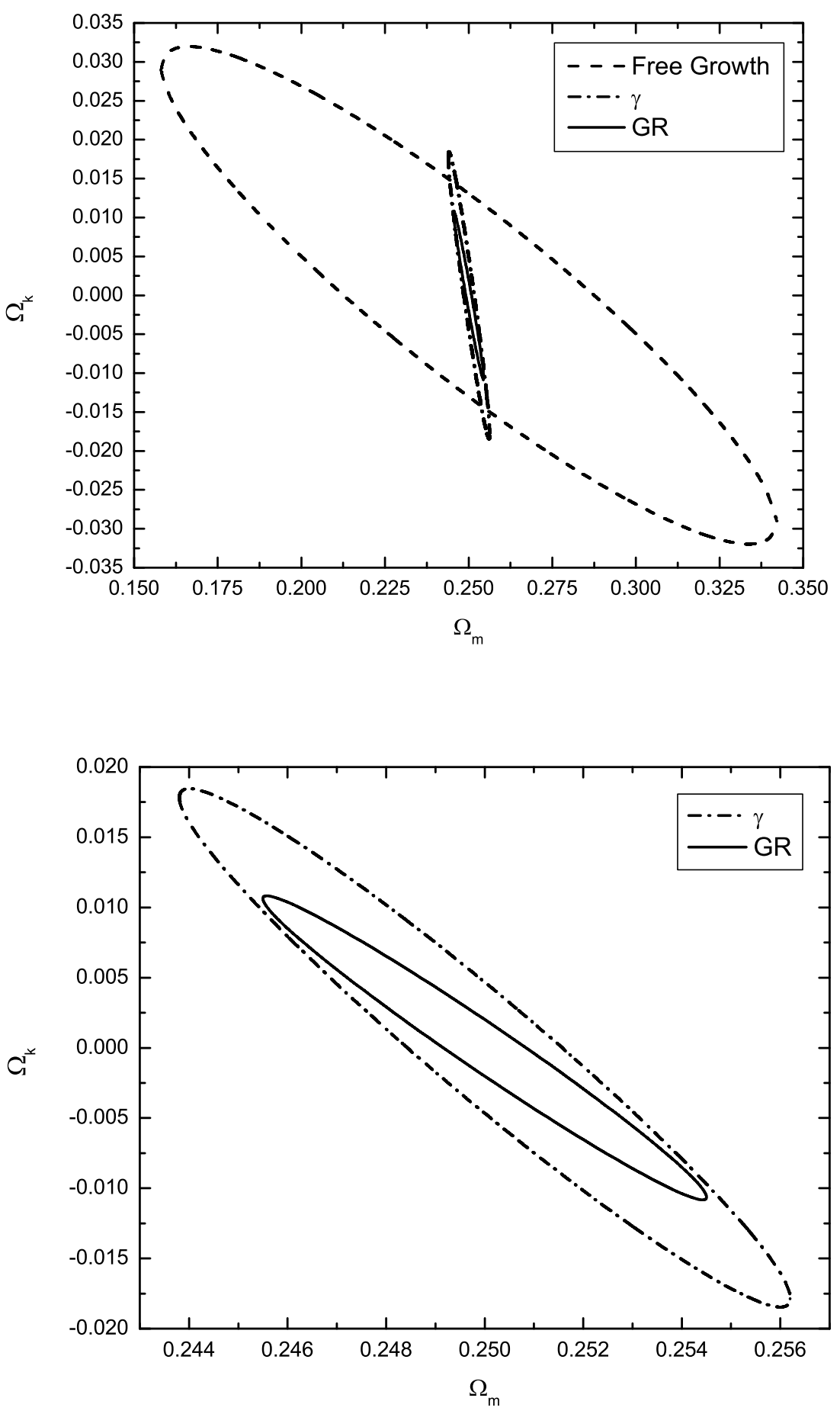

Fig. 6. - One standard deviation confidence level contour constraints on parameters $\Omega_{k}$ and $\Omega_{m}$ of the $\phi \mathrm{CDM}$ model. The lower panel shows a magnification of the two tightest contours in the center of the upper panel. 


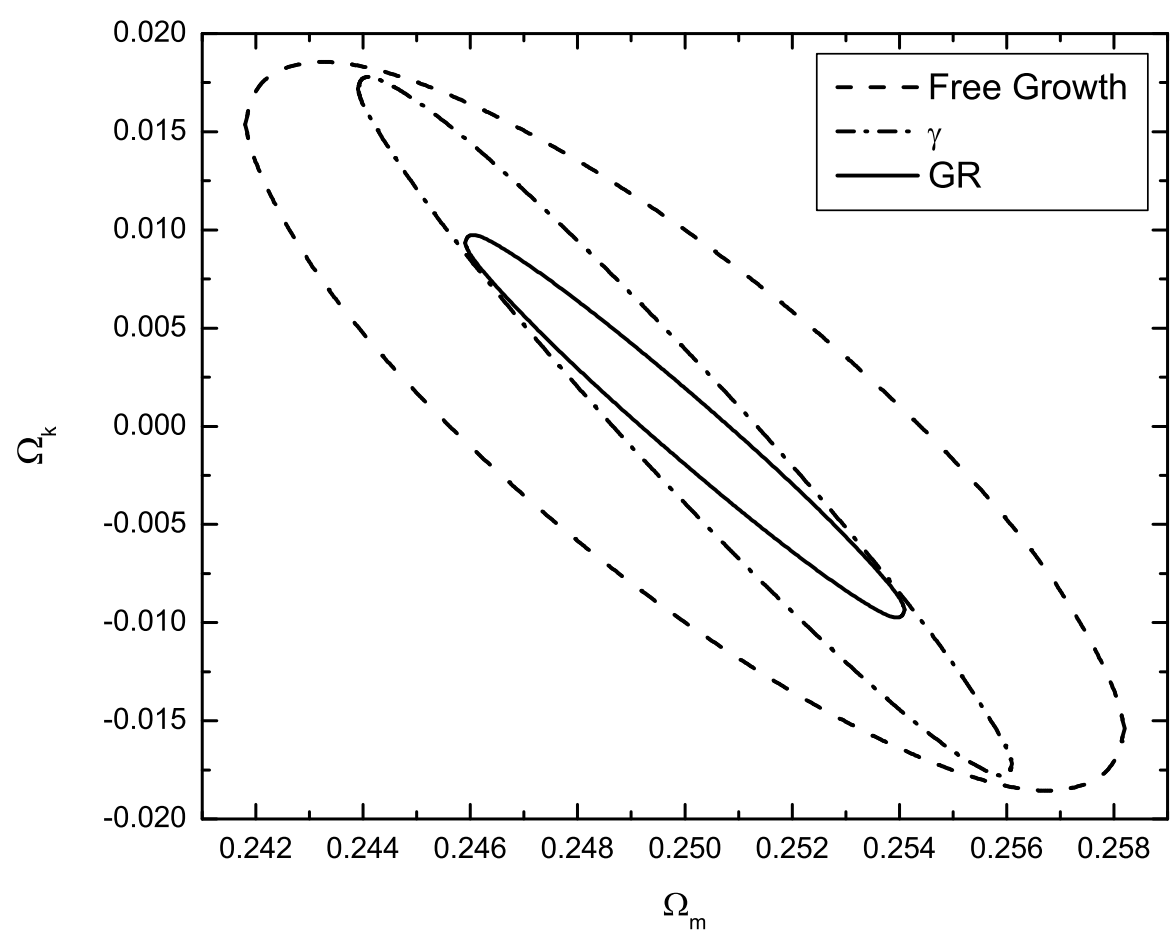

Fig. 7.- One standard deviation confidence level contour constraints on parameters $\Omega_{k}$ and $\Omega_{m}$ of the $\Lambda$ CDM model. 